\section{Electrical Potential Change Accompanying the Development of Rigor in lodoacetate- Poisoned Muscle}

No a priori answer can be given to the question whether the development of rigor in muscle poisoned by iodoacetate is accompanied by electrical depolarization, though it may be expected that the muscle after developing rigor will eventually become depolarized. The present work was an attempt to find an experimental answer to this question.

The sartorius muscle of the toad prepared with its nerve for stimulation was employed. The preparation was poisoned by soaking for 30-60 min. in $1 / 10,000-$ $1 / 20,000$ iodoacetate solution, sometimes with the addition of sodium cyanide to the concentration of $M / 1,000-2,000$. Rigor of the poisoned muscle was induced in the usual way by stimulation, at the rate of one shock every 5 sec. in most experiments. A rough indication that the development of rigor was not accompanied by any considerable depolarization was first obtained by showing that the muscle, soon after the development of rigor, gave demarcation potential of the usual magnitude on crushing or on application of isotonic potassium chloride.

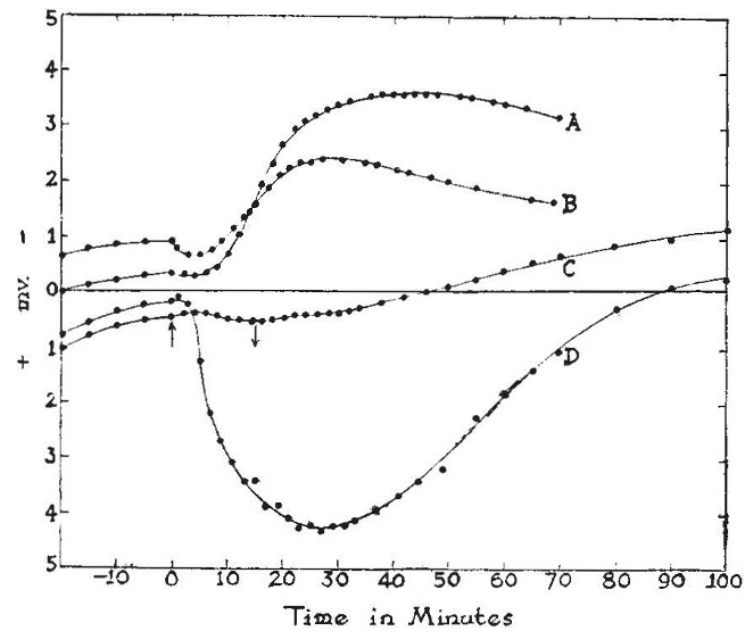

Time-course of electrical potential change of poisoned half of muscle relative to normal half. Stimulation, one shock every 5 sec. between arrows. See text for other explanation

A closer examination was then made by poisoning one half of the muscle and measuring the potential of this half against the normal half at short intervals during and after the onset of rigor. In such experiments the muscle was naturally mounted in air and the results regularly showed a slowly developing depolarization, beginning shortly after the start of stimulation and reaching a maximum in 30-40 min., roughly paralleling the course of the development of rigor. Both the course and the magnitude of the depolarization, however, depended on the temperature. The results of an experiment at $10-11^{\circ} \mathrm{C}$. are reproduced in the accompanying graph, curves $A$ and $B$, given by two symmetrical muscles. A comparison of pairs of curves such as these revealed the influence of an apparently insignificant difference in the experimental condition. When two strands of cotton-wool were used for connecting the muscle to the calomel half-cells used as leads (curve $A$ ), the depolarization was regularly greater than when two troughs about $8 \mathrm{~mm}$. wide filled with Ringer solution served as contacts (curve $B$ ). The only obvious difference was that, if there was anything liberated by the muscle, it would be partially washed away from the segments of the muscle lying on the troughs.

We therefore tested the effect of deliberate washing. It was found that the depolarization of the poisoned half relative to the normal half observed when the muscle was mounted and stimulated in air disappeared more or less completely when the whole muscle was afterwards washed in stirred normal Ringer solution. We then attempted to determine the time-course of the electrical potential change accompanying the development of rigor while the whole muscle, one half of which was previously poisoned, was continually washed except during the short times required for potential measurements. Curve $C$ represents the result when the washing solution was normal Ringer. It is seen that the depolarization of the poisoned half normally occurring during the first 30-40 min. after the beginning of stimulation was completely suppressed.

There is thus a strong indication that the initial depolarization accompanying the development of rigor was due to the release of some depolarizing substance by the muscle when developing rigor. In all probability this substance was potassium. Dean $^{1}$ has previously reported that iodoacetatepoisoned muscle, after the onset of rigor, lost a large amount of potassium. The use of potassium-free Ringer solution as a possibly more efficient washing solution then suggested itself, and the result was as shown in curve $D$, the two curves $C$ and $D$ being given by a symmetrical pair of muscles at $23^{\circ} \mathrm{C}$. treated in identical manner except for the composition of the washing solution, and therefore directly comparable.

It is seen that, under the conditions of continuous washing with potassium-free Ringer solution, the initial phase of depolarization may be not only com. pletely suppressed, but even actually replaced by a phase of large positive variation. That the half of the muscle subjected to rigor under these conditions contained less potassium than the normal half had been ascertained by separate potassium analysis of the two halves. A mere smaller potassium content of that half, however, could not account for its relative positivity. We had soaked half the muscle in potassium-free Ringer solution overnight, and then washed the whole muscle in potassium-free Ringer for half to one hour. The end which had previously been soaked in potassium-free Ringer solu. tion overnight, while containing less potassium as afterwards determined by analysis, showed no relative positivity.

It may be concluded that when secondary change resulting from the release of potassium is eliminated, the development of rigor in iodoacetate-poisoned muscle is not accompanied by depolarization. Indeed, the primary event might even be an increase of polarization.

Y. M. LIU

C. H. Hsu

T. P. FENG

Institute of Medicine, Academia Sinica, Shanghai. June 24.

${ }^{1}$ Dean, R. B., J. Cell. Comp. Physiol., 15, 189 (1940). 